\title{
Deviations of Capacitive and Inductive Loops in the Electrochemical Impedance of a Dissolving Iron Electrode
}

\author{
Masayuki Itagaki,* Akihiro TAYA,* Kunihiro Watanabe,* and Kazuhiko NodA** \\ *Department of Pure and Applied Chemistry, Faculty of Science and Technology, Tokyo University of Science, \\ Noda, Chiba 278-8510, Japan \\ **Corrosion Research Group, National Institute for Materials Science, \\ Sengen, Tsukuba, Ibaraki 305-0047, Japan
}

\begin{abstract}
The electrochemical impedance of an iron electrode often shows the capacitive and inductive loops on the complex plane. The capacitive loop originates from the time constant of the charge transfer resistance and the electric double layer capacitance. The inductive loop is explained by Faradaic processes involving the reaction intermediate. In some cases, these loops deviate from a true semicircle. In this paper, the origins and curve-fitting methods for the deviated loops of electrochemical impedance are discussed. The constant phase element (CPE) was used to present the deviation of the capacitive loop instead of electric double layer capacitance. The reaction rate constants, which are a function of the frequency, are proposed for the Faradaic impedance to present the deviated inductive loop.
\end{abstract}

(Received January 9, 2002; Accepted March 28, 2002)

\section{Introduction}

Electrochemical impedance spectroscopy (EIS) has been applied to investigations of corrosion and other electrochemical systems. Typical electrochemical impedance describes a semicircle in the fourth quadrant (the real and imaginary parts are positive and negative, respectively) of the complex plane presented in Fig. 1(a). The semicircle in the fourth quadrant is called a capacitive loop in the analysis of EIS. The simple equivalent circuit for an electrode/solution interface is shown in Fig. 2(a), where $R_{\mathrm{ct}}$ is the charge transfer resistance, $C_{\mathrm{dl}}$ is the capacitance of the electric double layer and $R_{\text {sol }}$ is the solution resistance. The electrochemical reaction can be investigated by these parameters obtained by curve fitting using the equivalent circuit in Fig. 2. Among the experimental results of EIS, however, the center of the capacitive loop deviates from the real axis, and the loop diverges from a true semicircle. This deviation originates from the frequency dispersion of the capacitance by dielectric relaxation, and is interpreted by an equivalent circuit involving CPE (constant phase element) in Fig. 2(b). ${ }^{1}$ The mathematical expression for CPE is explained later in this article. Except for the above-mentioned deviation, the time variation and current distribution yield the divergences in the low and high frequency ranges, respectively. ${ }^{2-4}$

The electrochemical impedance of an iron electrode in an aqueous solution often shows not only a capacitive loop, but also an inductive loop, which is a semicircle in the first quadrant (both real and imaginary parts are positive), like as presented in Fig. 1(b). This impedance is presented by an equivalent circuit involving the Faradaic impedance $\left(Z_{\mathrm{F}}\right)$ in Fig. 2(c). In some cases, both the capacitive and inductive loops show the deviation depicted by the dashed lines in Fig. 1(b). In this paper, the reasons for these deviations are discussed. The deviation of the capacitive loop will be explained by the CPE.
On the other hand, since the inductive loop is represented by the Faradaic impedance, the diversion of the inductive loop cannot be analyzed by the CPE. Therefore, a new interpretation of the Faradaic impedance is proposed, while assuming the frequency dispersion of the elementary steps in the dissolution.

\section{Experimental}

The surface of the working electrode was a circle, which is the cross section of an $\mathrm{Fe}$ rod embedded in epoxy resin. The surface area of the working electrode was $3.1 \times 10^{-2} \mathrm{~cm}^{2}$. The
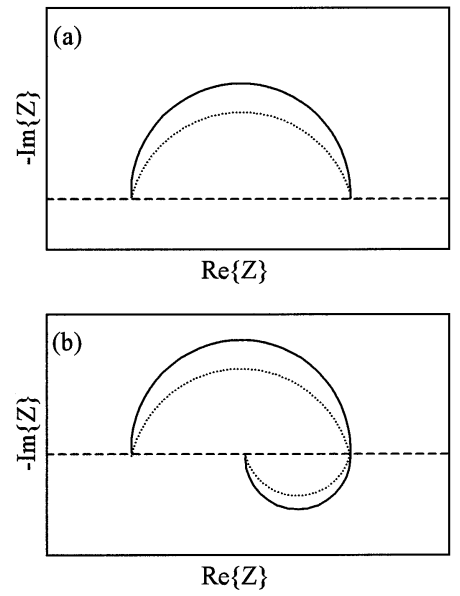

Fig. 1 Sheme of the electrochemical impedance on the complex plane. (a) Impedance showing capacitive loop. (b) Impedance showing capacitive and inductive loops. The line indicates a perfect semicircle. The dashed line indicates a diverged semicircle. 

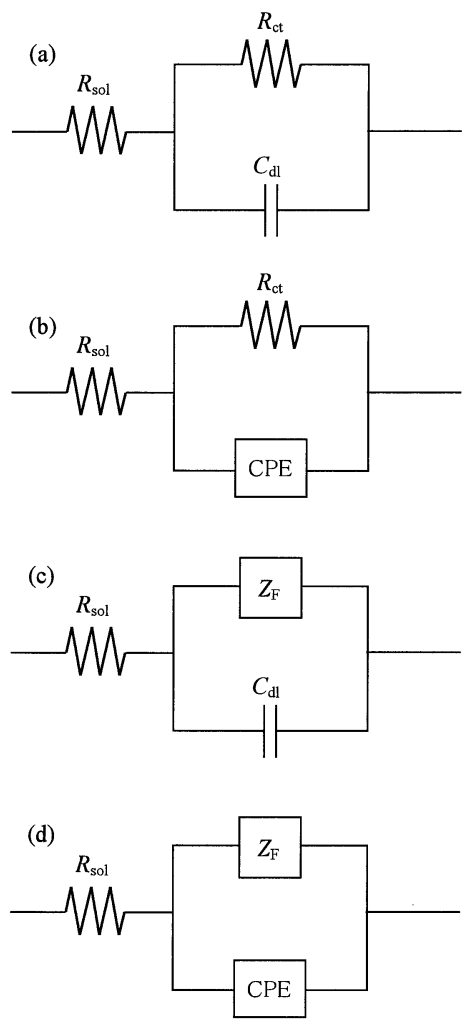

Fig. 2 Equivalent circuits for an electrode/solution interface. (a) Simplest circuit. $R_{\mathrm{ct}}$ is the charge transfer resistance. $C_{\mathrm{dl}}$ is the capacitance of an electric double layer. $R_{\text {sol }}$ is the solution resistance. (b) Circuit with CPE. (c) Circuit with Faradaic impedance $\left(Z_{\mathrm{F}}\right)$. (d) Circuit with $\mathrm{CPE}$ and $Z_{\mathrm{F}}$.

working electrode was polished by emery paper (\#2000) and rinsed by methanol and distilled water before a measurement. The counter and reference electrodes were $\mathrm{Pt}$ and saturated $\mathrm{KCl} / \mathrm{AgCl} / \mathrm{Ag}$, respectively.

The electrolyte solution was a mixture of $0.5 \mathrm{~mol} / \mathrm{dm}^{3} \mathrm{H}_{2} \mathrm{SO}_{4}$ and $0.5 \mathrm{~mol} / \mathrm{dm}^{3} \mathrm{Na}_{2} \mathrm{SO}_{4}$ containing an arbitrary concentration of $\mathrm{NaBr}$, and adjusted at $\mathrm{pH} 1$. The electrolyte solution was prepared by analytical grade chemicals and double-distilled water, and deaerated by the bubbling of high purity nitrogen gas.

The electrochemical impedances were measured by using a potentio/galvanostat (Hokuto, HA501G) and a frequency response analyzer (NFblock, FRA5020). The experimental instruments were controlled by a desktop computer via a GPIB interface. The amplitude of the imposed potential modulation was $10 \mathrm{mV}$, and the frequency range was from $10 \mathrm{mHz}$ to 10 $\mathrm{kHz}$ in measuring the electrochemical impedance.

\section{Results and Discussion}

Faradaic impedance of the dissolving metal electrode

When the electrode reaction involves only simple charge transfer, the electrode resistance is represented by $R_{\mathrm{ct}}$. In this case, the electrochemical impedance is analyzed by the equivalent circuit shown in Fig. 2(a), and describes the capacitive loop. When the metal dissolution involves a reaction intermediate, the electrode resistance has the time constant in the dissolution process and is expressed as the Faradaic impedance $\left(Z_{\mathrm{F}}\right)$, which is the frequency-dependent resistance related to a complicated electrode reaction. ${ }^{5}$ Epelboin et al. ${ }^{6}$ derived the $Z_{\mathrm{F}}$ of the iron electrode in an acidic solution as follows. The metal (M) is oxidized to $\mathrm{M}$ (II) via an adsorbed intermediate $\left(\mathrm{M}(\mathrm{I})_{\mathrm{ad}}\right)$ :

$$
\begin{aligned}
& \mathrm{M} \stackrel{K_{1}}{\longrightarrow} \mathrm{M}(\mathrm{I})_{\mathrm{ad}}+\mathrm{e}^{-}, \\
& \mathrm{M}(\mathrm{I})_{\mathrm{ad}} \stackrel{K_{2}}{\longrightarrow} \mathrm{M}(\mathrm{II})+\mathrm{e}^{-},
\end{aligned}
$$

where $K_{\mathrm{i}}$ is potential-dependent rate constant $\left(K_{\mathrm{i}}=k_{\mathrm{i}} \exp \left(b_{\mathrm{i}} E\right)\right.$, where $k_{\mathrm{i}}$ is the rate constant at $E=0$ and $b_{\mathrm{i}}$ is the Tafel constant). From these reactions, assuming that they obey to the Langmuir isotherm, the mass balance of $\mathrm{M}(\mathrm{I})_{\text {ad }}$ and the current $i$ are written as:

$$
\begin{aligned}
& \gamma \frac{\mathrm{d} \theta}{\mathrm{d} t}=K_{1}(1-\theta)-K_{2} \theta, \\
& i=F\left\{K_{1}(1-\theta)+K_{2} \theta\right\},
\end{aligned}
$$

where $\theta$ is the fractional coverage of $\mathrm{M}(\mathrm{I})_{\mathrm{ad}}, \gamma$ the maximum surface concentration of $\mathrm{M}(\mathrm{I})_{\mathrm{ad}}\left(\mathrm{mol} / \mathrm{cm}^{2}\right)$, and $F$ the Faraday constant. Since $\mathrm{d} \theta / \mathrm{d} t=0$ at the steady-state, the steady-state coverage $(\Theta)$ is calculated from Eq. (3) as:

$$
\Theta=\frac{K_{1}}{K_{1}+K_{2}} .
$$

Moreover, the following equations are derived from Eqs. (3) and (4) by Fourier transformation and Taylor series expansion:

$$
\begin{aligned}
& j \omega \gamma \Delta \theta=\left\{b_{1} K_{1}(1-\Theta)\right\} \Delta E-\left(K_{1}+K_{2}\right) \Delta \theta, \\
& \frac{\Delta \theta}{\Delta E}=\frac{b_{1} K_{1}(1-\Theta)-b_{2} K_{2} \Theta}{j \omega \gamma+K_{1}+K_{2}}, \\
& \frac{\Delta i}{F}=\left\{b_{1} K_{1}(1-\Theta)+b_{2} K_{2} \Theta\right\} \Delta E+\left(K_{2}-K_{1}\right) \Delta \theta, \\
& \frac{\Delta i}{\Delta E}=\frac{K_{2}-K_{1}}{F} \frac{\Delta \theta}{\Delta E}+\frac{b_{1} K_{1}(1-\Theta)+b_{2} K_{2} \Theta}{F} .
\end{aligned}
$$

In Eq. (6), $j$ is an imaginary number and $\omega$ is the angular frequency. The Faradaic impedance $\left(Z_{\mathrm{F}}\right)$ is obtained from the reciprocal of the admittance $(\Delta i / \Delta E)$ in Eq. (9),

$$
Z_{\mathrm{F}}=\frac{F}{\left(K_{2}-K_{1}\right) \frac{\Delta \theta}{\Delta E}+b_{1} K_{1}(1-\Theta)+b_{2} K_{2} \Theta}
$$

The $Z_{\mathrm{F}}$ expressed by Eq. (10) describes the inductive loop in Fig. 1(b), while selecting suitable kinetic parameters. The causes of the deviation shown by the dashed lines in Fig. 1 are discussed in the following sections.

\section{Deviation of the capacitive loop}

Dielectric relaxation is a phenomenon in which the electric current density follows the change of an electric field with a delay. In the case of dielectric loss, the permittivity is a function of the frequency, and is represented by a complex number. Moreover, an electric double layer cannot be treated by a simple capacitance in the EIS measurement due to the dielectric loss, and the capacitive loop shows the deviation given in Fig. 1(a). In this case, the electric double layer is regarded by $\mathrm{CPE},{ }^{1}$ and its admittance $\left(Y_{\mathrm{CPE}}\right)$ is written as: 


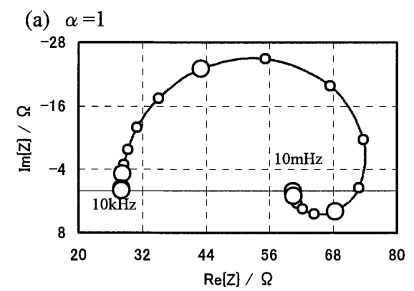

(c) $\alpha=0.8$
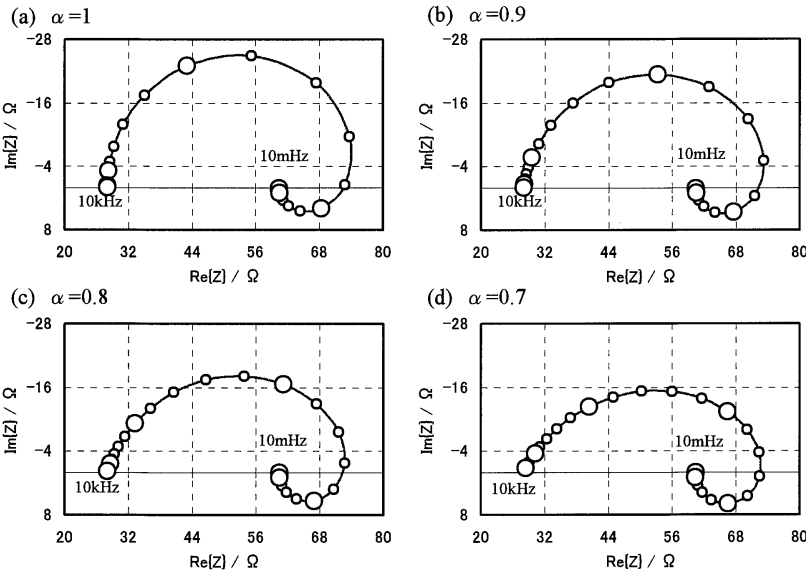

(d) $\alpha=0.7$

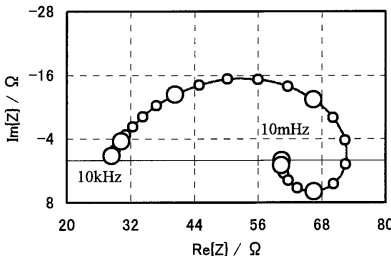

Fig. 3 Impedances calculated by the equivalent circuit in Fig. 2(d), $R_{\text {sol }}=28 \Omega \mathrm{cm}^{3}, Q=5 \times 10^{-4} \Omega^{-1} \mathrm{~cm}^{-2} \mathrm{~s}^{\alpha}, b_{1}=38 \mathrm{~V}^{-1}, b_{2}=7 \mathrm{~V}^{-1}, K_{1}=$ $5 \times 10^{-9} \mathrm{~mol} \mathrm{~s}^{-1} \mathrm{~cm}^{-2}, K_{2}=5 \times 10^{-8} \mathrm{~mol} \mathrm{~s}^{-1} \mathrm{~cm}^{-2}, \gamma=1 \times 10^{-8} \mathrm{~mol}$ $\mathrm{cm}^{-2}$. (a) $\alpha=1$, (b) $\alpha=0.9$, (c) $\alpha=0.8$, (d) $\alpha=0.7$.

$$
Y_{\mathrm{CPE}}=Q(j \omega)^{\alpha},
$$

where $Q$ and $\alpha$ are CPE parameters, and $\alpha$ takes a value between -1 and 1 . When $Q=C_{\mathrm{dl}}$ and $\alpha=1, Y_{\mathrm{CPE}}$ is the simple admittance of the electric double layer $\left(Y_{\mathrm{CPE}}=j \omega C_{\mathrm{dl}}\right)$. Figure 2(d) shows an equivalent circuit involving CPE and $Z_{\mathrm{F}}$, and the impedance $(Z)$ is given as:

$$
Z=R_{\mathrm{sol}}+\left(\frac{1}{Z_{\mathrm{F}}}+Y_{\mathrm{CPE}}\right)^{-1}
$$

Figure 3 shows the results calculated by Eq. (12). The capacitive and inductive loops are described on the complex plane. It is shown that the deviation of the capacitive loop becomes significant with a decrease of $\alpha$.

\section{Deviation of the inductive loop}

The reason why the inductive loop deviates from a true semicircle is discussed while assuming that the rate constants is a function of the frequency:

$$
K_{\mathrm{i}}=K_{\mathrm{i}}^{\prime}(j \omega)^{\beta},
$$

where $\beta$ is a parameter used to present the phase shift. In Eq. (13), $\beta>0$ means that the reaction rate proceeds to the imposed sinusoidal potential modulation. Contrary to this, $\beta<$ means the delay of the phase shift. Though the rate constant is generally independent on the frequency, it is considered that reactions (1) and (2) have the additional elementary steps. The physical meanings in this assumption are as follows: For $\beta>0$, the reaction rate decays after potential modulation. For $\beta<0$, the reaction shows an induction period against potential modulation.

From reactions (1), (2) and Eq. (13), the mass balance of $\mathrm{M}(\mathrm{I})_{\mathrm{ad}}$ and the current are

$$
\begin{aligned}
& \gamma \frac{\mathrm{d} \theta}{\mathrm{d} t}=K_{1}^{\prime}(j \omega)^{\beta}(1-\theta)-K_{2}^{\prime}(j \omega)^{\beta} \theta, \\
& i=F(j \omega)^{\beta}\left\{K_{1}^{\prime}(1-\theta)+K_{2}^{\prime} \theta\right\} .
\end{aligned}
$$

The Faradaic impedance $\left(Z_{\mathrm{F}}\right)$ and electrode impedance $(Z)$ are determined as follows:
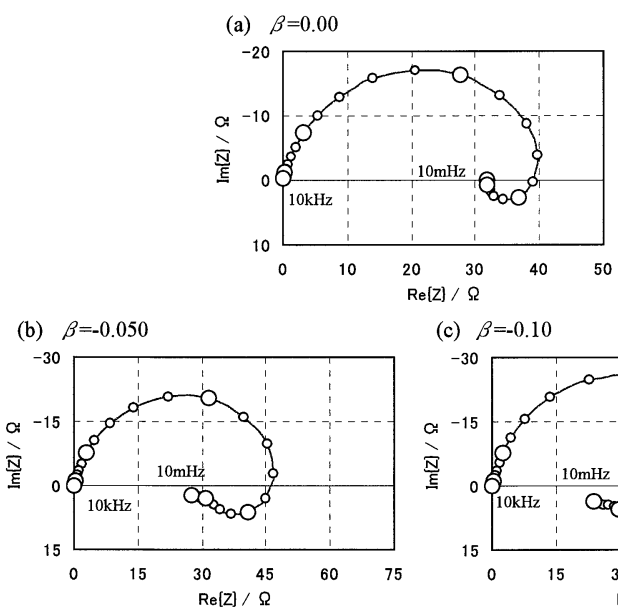

(c) $\beta=-0.10$
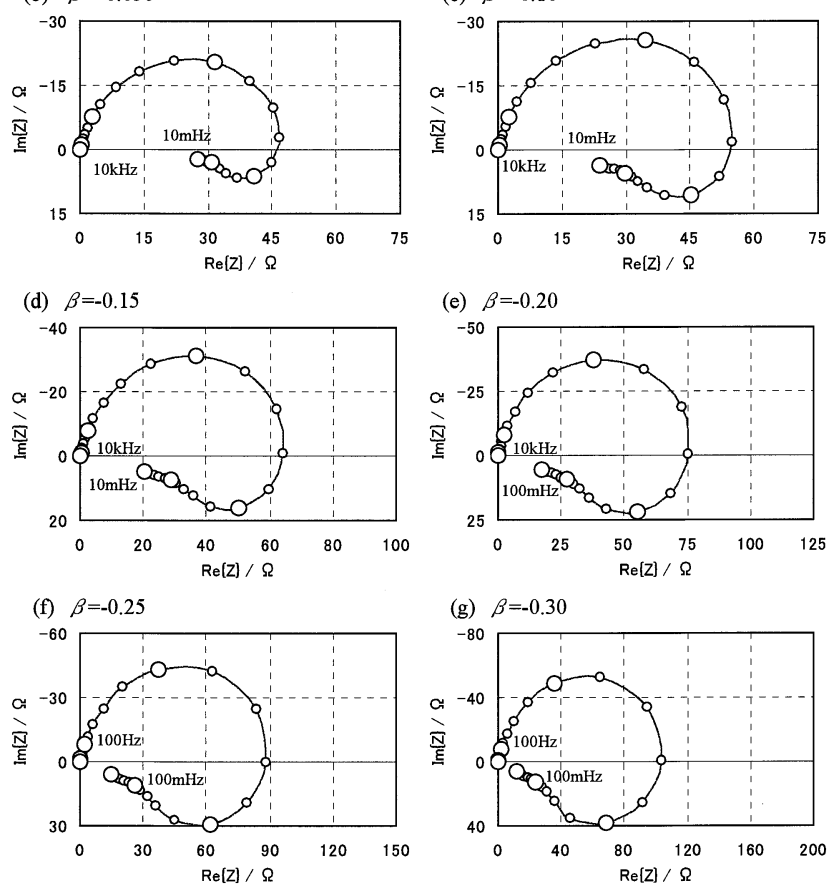

Fig. 4 Impedances calculated by the equivalent in Fig. 2(d) when $K_{\mathrm{i}}=K_{\mathrm{i}}^{\prime}(j \omega)^{\beta} . \quad R_{\mathrm{sol}}=0 \Omega \mathrm{cm}^{2}, b_{1}=38 \mathrm{~V}^{-1}, b_{2}=7 \mathrm{~V}^{-1}, K_{1}^{\prime}=5 \times 10^{-9}$ $\mathrm{mol} \mathrm{s}-1 \mathrm{~cm}^{-2}, K_{2}^{\prime}=5 \times 10^{-8} \mathrm{~mol} \mathrm{~s}^{-1} \mathrm{~cm}^{-2}, \gamma=1 \times 10^{-8} \mathrm{~mol} \mathrm{~cm}^{-2}$. (a) $\beta$ $=0$, (b) $\beta=-0.05$, (c) $\beta=-0.1$, (d) $\beta=-0.15$, (e) $\beta=-0.2$, (f) $\beta=$ -0.25 , (g) $\beta=-0.3$.

$$
j \omega \gamma \Delta \theta=(j \omega)^{\beta}\left[\left\{b_{1} K_{1}^{\prime}-\left(b_{1} K_{1}^{\prime}+b_{2} K_{2}^{\prime}\right) \Theta\right\} \Delta E-\left(K_{1}^{\prime}+K_{2}^{\prime}\right) \Delta \theta\right],(16)
$$

$$
Z=R_{\mathrm{sol}}+\left(\frac{1}{Z_{\mathrm{F}}}+j \omega C_{\mathrm{dl}}\right)^{-1}
$$

Figures 4 and 5 show $Z$ at various value of $\beta . \quad K_{\mathrm{i}}$ is independent of the frequency when $\beta=0$, and $Z$ describes a perfect capacitive and the inductive semicircles in Fig. 4. When $\beta<0$ in Fig. 4, the inductive loop due to the Faradaic impedance shows a deviation. This deviation becomes significant and the diameter of the loop increases with a decrease of $\beta$. When $\beta>0$ in Fig. 5, not only inductive loop, but also capacitive loop, deviate from a semicircle.

\section{Comparison of calculations with the experimental results}

Figures 6(a) and (b) show the electrochemical impedances ${ }^{7}$ of iron in a sulfuric acid solution. The capacitive and inductive loops are observed in these impedances. The capacitive loop shows a deviation. This deviation was also observed in the electrochemical impedance which Keddam et al. ${ }^{6,8}$ measured by an iron electrode in a sulfuric acid solution. Lorenz et al. ${ }^{9}$ estimated the change transfer resistance of an iron electrode in a sulfuric acid solution by curve fitting using a Cole-Cole plot. ${ }^{10}$ Lorenz et al. revealed in this paper ${ }^{9}$ that the Cole-Cole plot is available for a capacitive loop, but not for a deviated inductive 

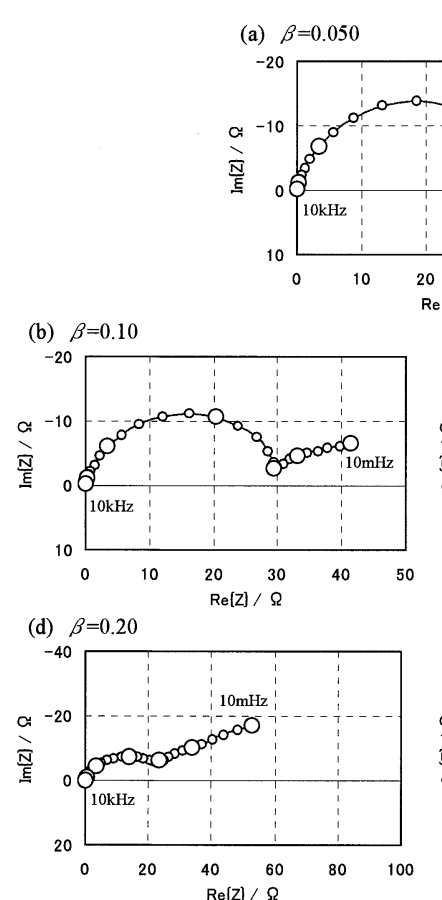

(f) $\beta=0.30$
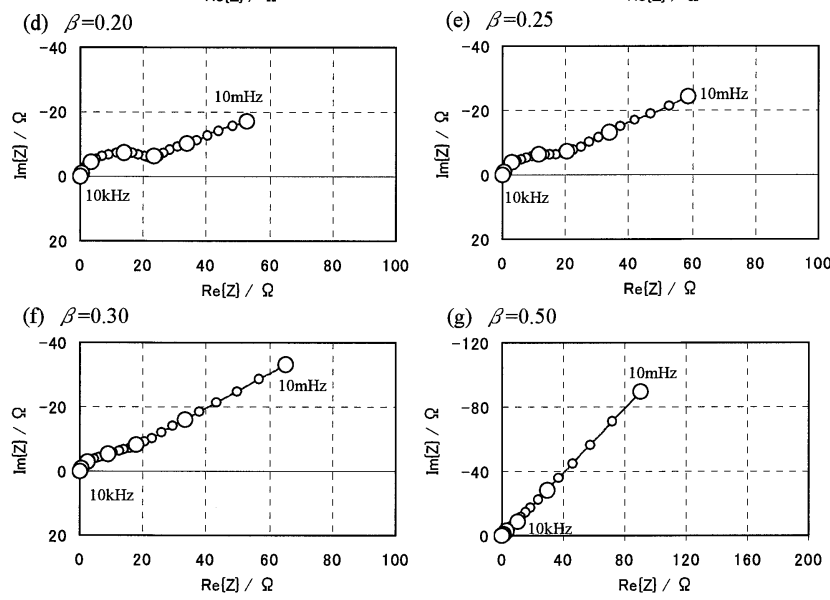

Fig. 5 Impedances calculated by the equivalent in Fig. 2(d) when $K_{\mathrm{i}}=K_{\mathrm{i}}^{\prime}(j \omega)^{\beta} . R_{\text {sol }}=0 \Omega \mathrm{cm}^{2}, b_{1}=38 \mathrm{~V}^{-1}, b_{2}=7 \mathrm{~V}^{-1}, K_{1}^{\prime}=5 \times 10^{-9}$ $\mathrm{mol} \mathrm{s}{ }^{-1} \mathrm{~cm}^{-2}, K_{2}^{\prime}=5 \times 10^{-8} \mathrm{~mol} \mathrm{~s}^{-1} \mathrm{~cm}^{-2}, \gamma=1 \times 10^{-8} \mathrm{~mol} \mathrm{~cm}^{-2}$. (a) $\beta$ $=0.05$, (b) $\beta=0.1$, (c) $\beta=0.15$, (d) $\beta=0.2$, (e) $\beta=0.25$, (f) $\beta=0.3$, (g) $\beta=0.5$.

loop. Figures 6(c) and (d) show the $Z$ of iron in a sulfuric acid solution containing $\mathrm{NaBr} .{ }^{11}$ The ratio of the diameter of inductive loop to the capacitive loop in Figs. 6(c) and (d) is larger than that in Figs. 6(a) and (b). It is well known that the inductive behavior becomes remarkable for the $Z$ of iron in a sulfuric solution containing $\mathrm{NaBr}, \mathrm{NaI}^{11}$ and Propargylic alcohol. ${ }^{12}$ All impedances in Fig. 6 were simulated by the equivalent circuit in Fig. 2(d), and the simulated results are presented by the line in Fig. 6 . The value of $\beta$ is between -0.03 and -0.06 , indicating that iron dissolves by a consecutive dissolution mechanism, and that reactions (1) and (2) involve a delay to the imposed potential modulation. In this paper, the details of the elementary steps in reactions (1) and (2) are difficult to clarify, though the deviated capacitive and inductive loops can be simulated geometrically using the CPE and $Z_{\mathrm{F}}$ derived in section 3.3. This curve-fitting method contributes to the analysis of an impedance that shows an inductive loop, because perfect curve fitting had been impossible for a diverged
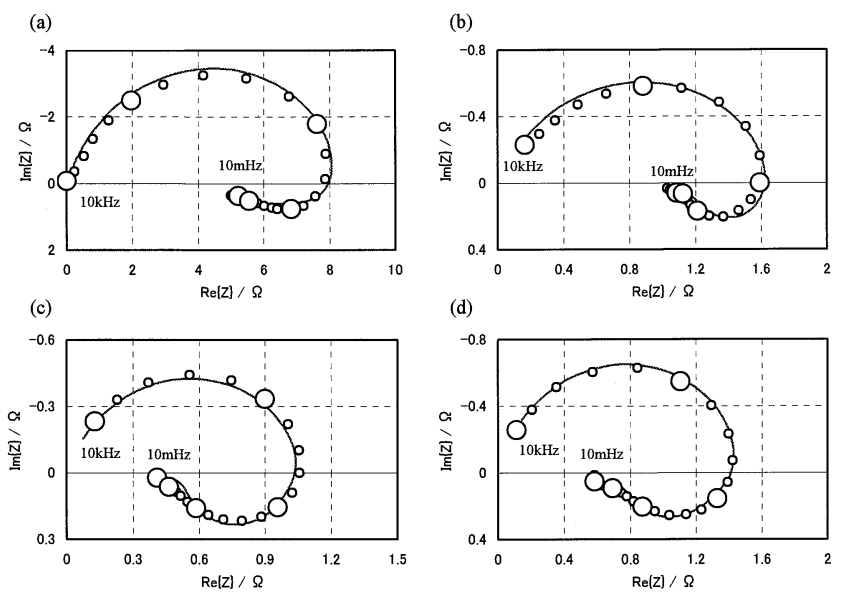

Fig. 6 Electrochemical impedance of an iron electrode. The plots mean the experimental results. The lines mean simulated results. The experimental conditions and the values of $\alpha$ and $\beta$ are as follows: (a) Solution, sulfuric acid ( $\mathrm{pH}$ 1); electrode potential, $-0.45 \mathrm{~V} ; \alpha=$ $0.80 ; \beta=-0.05 ; R_{\text {sol }}=0 \Omega \mathrm{cm}^{2} ; b_{1}=38 \mathrm{~V}^{-1} ; b_{2}=7 \mathrm{~V}^{-1} ; K_{1}^{\prime}=7.8 \times$ $10^{-8} \mathrm{~mol} \mathrm{~s}^{-1} \mathrm{~cm}^{-2} ; K_{2}^{\prime}=7.5 \times 10^{-8} \mathrm{~mol} \mathrm{~s}^{-1} \mathrm{~cm}^{-2} ; \gamma=1 \times 10^{-8} \mathrm{~mol} \mathrm{~cm}{ }^{-2}$. (b) Solution, sulfuric acid (pH 1); electrode potential, $-0.40 \mathrm{~V} ; \alpha=$ $0.75 ; \beta=-0.03, R_{\text {sol }}=0 \Omega \mathrm{cm}^{2} ; b_{1}=38 \mathrm{~V}^{-1} ; b_{2}=7 \mathrm{~V}^{-1} ; K_{1}^{\prime}=2.3 \times$ $10^{-7} \mathrm{~mol} \mathrm{~s}^{-1} \mathrm{~cm}^{-2} ; K_{2}^{\prime}=5.5 \times 10^{-7} \mathrm{~mol} \mathrm{~s}^{-1} \mathrm{~cm}^{-2} ; \gamma=1 \times 10^{-8} \mathrm{~mol} \mathrm{~cm}{ }^{-2}$. (c) Solution, sulfuric acid (pH 1) containing $0.3 \mathrm{~mol} \mathrm{dm}^{-3} \mathrm{NaBr}$; electrode potential, $-0.30 \mathrm{~V} ; \alpha=0.80 ; \beta=-0.04 ; R_{\text {sol }}=0 \Omega \mathrm{cm}^{2}, b_{1}=$ $38 \mathrm{~V}^{-1} ; b_{2}=7 \mathrm{~V}^{-1} ; K_{1}^{\prime}=2.9 \times 10^{-7} \mathrm{~mol} \mathrm{~s}^{-1} \mathrm{~cm}^{-2} ; K_{2}^{\prime}=5 \times 10^{-6} \mathrm{~mol} \mathrm{~s}^{-1}$ $\mathrm{cm}^{-2} ; \gamma=5 \times 10^{-7} \mathrm{~mol} \mathrm{~cm}^{-2}$. (d) Solution, sulfuric acid (pH 1) containing $1 \mathrm{~mol} \mathrm{dm}{ }^{-3} \mathrm{NaBr}$; electrode potential, $-0.30 \mathrm{~V} ; \alpha=0.85$, $\beta=-0.06 ; R_{\text {sol }}=0 \Omega \mathrm{cm}^{2} ; b_{1}=38 \mathrm{~V}^{-1} ; b_{2}=7 \mathrm{~V}^{-1}, K_{1}^{\prime}=3.1 \times 10^{-7} \mathrm{~mol}$ $\mathrm{s}^{-1} \mathrm{~cm}^{-2} ; K_{2}^{\prime}=1 \times 10^{-6} \mathrm{~mol} \mathrm{~s}^{-1} \mathrm{~cm}^{-2} ; \gamma=5 \times 10^{-8} \mathrm{~mol} \mathrm{~cm}^{-2}$.

inductive loop until the present paper

\section{References}

1. P. Zoltowski, J. Electroanal. Chem., 1998, 443, 149.

2. Z. Stoynov, Electrochim. Acta, 1993, 38, 1919.

3. M. Itagaki, A. Taya, and K. Watanabe, Electrochemistry, 2000, 68, 596.

4. R. de Levie, Electrochim. Acta, 1964, 9, 1231.

5. M. Itagaki and K. Watanabe, Denki Kagaku, 1997, 65, 758.

6. I. Epelboin and M. Keddam, J. Electrochem. Soc., 1970, 117, 1052.

7. M. Itagaki, M. Tagaki, and K. Watanabe, Electrochim. Acta, 1996, 41, 1201.

8. M. Keddam, O. R. Mattos, and H. Takenouti, J. Electrochem. Soc., 1981, 128, 257.

9. W. J. Lorenz and F. Mansteld, Corros. Sci., 1981, 21, 647.

10. K. S. Cole and R. H. Cole, J. Chem. Phys., 1941, 9, 341; and 1942, 10, 98.

11. M. Itagaki, M. Tagaki, and K. Watanabe, Denki Kagaku, 1995, 63, 808 .

12. I. Epelboin, M. Keddam, and H. Takenouti, J. Appl. Electrochem., 1972, 2, 71. 